\title{
Multicenter, randomized, tandem evaluation of EndoRings colonoscopy - results of the CLEVER study
}

Authors

Institutions
Vincent K. Dik ${ }^{1}$, Ian M. Gralnek ${ }^{2,3,4}$, Ori Segol ${ }^{3,5}$, Alain Suissa ${ }^{3,6}$, Tim D. G. Belderbos ${ }^{1}$, Leon M. G. Moons ${ }^{1}$, Meytal Segev $^{7}$, Sveta Domanov ${ }^{3}$, Douglas K. Rex ${ }^{8}$, Peter D. Siersema ${ }^{1}$

Institutions are listed at end of article. submitted 24. January 2015 accepted after revision 29. April 2015

\section{Bibliography Dol http://dx.doi.org/ 10.1055/s-0034-1392421 Published online: 28.7 .2015 Endoscopy 2015; 47: 1151- 1158 \\ (c) Georg Thieme Verlag KG Stuttgart · New York ISSN 0013-726X}

\section{Corresponding author} Ian M. Gralnek, MD, MSHS Institute of Gastroenterology and Liver Diseases Ha’Emek Medical Center Afula 18101 Israel ian_gr@clalit.org.il
Background and study aims: Adenoma miss rate during colonoscopy has become a widely acknowledged proxy measure for post-colonoscopy colorectal cancer. Among other reasons, this can happen because of inadequate visualization of the proximal aspects of colonic folds and flexures. EndoRings (EndoAid Ltd., Caesarea, Israel) is a silicone-rubber device that is fitted onto the distal end of the colonoscope. Its flexible circular rings engage and mechanically stretch colonic folds during withdrawal. The primary aim of this study was to compare adenoma miss rates between standard colonoscopy and colonoscopy using EndoRings.

Methods: In this multicenter, randomized, tandem colonoscopy study, we performed same-day, back-to-back colonoscopies with EndoRings followed by standard colonoscopy, or vice versa.

Results: After exclusion of 10 patients for protocol violations, 116 patients (38.8\% female; mean age 58.7) remained for analysis. The adenoma miss

\section{Introduction $\nabla$}

Colonoscopy is the standard method for detection and removal of colorectal adenomas, the precursor lesions of most colorectal cancers [1-4]. Despite being the most sensitive method, colonoscopy does not fully protect a person from developing future colorectal cancer [5-10]. This imperfection is for a major part caused by missed precancerous lesions due to inadequate bowel preparation, the presence of flat lesions that are difficult to detect $[8,9,11]$, and the relative difficulty of visualizing lesions on the proximal side of haustral folds and the internal curves of flexures $[12,13]$. The latter may, at least partly, be caused by the fact that the use of standard $140^{\circ}$ and $170^{\circ}$ colonoscopes allows visualization of only approximately $90 \%$ of the colonic surface [14]. Moreover, it has been shown that up to two- rate of EndoRings colonoscopy $(7 / 67 ; 10.4 \%)$ was significantly lower $(P<0.001)$ compared with standard colonoscopy (28/58; $48.3 \%)$. Similar results were found for polyp miss rates: EndoRings (9.1\%) and standard colonoscopy (52.8\%; $P<$ 0.001 ). Mean cecal intubation times (9.3 vs. 8.4 minutes; $P=0.142$ ) and withdrawal times (7.4 vs. 7.2 minutes; $P=0.286$ ), respectively, were not significantly different between EndoRings and standard colonoscopy. Mean total procedure time was longer with EndoRings than with standard colonoscopy (21.6 vs. 18.5 minutes, $P=0.001$ ) as more polyps were removed.

Conclusions: This study demonstrates that colonoscopy with EndoRings has lower adenoma and polyp miss rates than standard colonoscopy, which may improve the efficacy particularly of screening and surveillance colonoscopies.

ClinicalTrials.gov NCT01955122.

thirds of missed lesions are located on the proximal side of folds [13].

The miss rates of colonoscopy have been widely acknowledged, with back-to-back colonoscopy studies showing polyp and adenoma miss rates of approximately $20 \%-25 \%$ in older studies [15] and up to $40 \%$ in more recent studies evaluating novel technologies developed to improve visualization behind folds $[16,17]$. Alongside the implementation of quality indicators $[1,18]$, technologies such as cap-assisted colonoscopy, virtual chromoendoscopy, Third Eye colonoscopy, and Full Spectrum Endoscopy (Fuse) colonoscopy have been developed to improve adenoma detection $[16,17,19,20]$. However, some of these techniques have been shown to increase only marginally the detection of adenomas, while others are less practical in use or demand high investments with a change in endoscopy platform $[21,22]$. 
In order to optimize the efficacy of colonoscopy, easy-to-use technological developments that aim to improve colonic visualization while maintaining standard colonoscopic capabilities and keeping the procedural time and associated costs to a minimum are required. One such device is the EndoRings (EndoAid Ltd., Caesarea, Israel), which is a silicone-rubber device that is fitted onto the distal end of the colonoscope ( Fig. 1). Its flexible circular rings allow cecal intubation but mechanically stretch the colonic folds during withdrawal and keep the tip of the colonoscope centered within the colonic lumen.

In this study we aimed to investigate the diagnostic yield of colonoscopy with EndoRings as compared with standard colonoscopy by comparing the adenoma and polyp miss rates of both techniques. We further compared adenoma and polyp detection rates; impact on colonoscopy surveillance intervals; times for cecal intubation, withdrawal, and the total procedure; and adverse events.

\section{Patients and methods \\ $\nabla$}

\section{Study design}

For this randomized, multicenter, tandem colonoscopy study we enrolled patients aged between 40 and 75 years who were referred for screening, surveillance, or diagnostic colonoscopy. Exclusion criteria were a history of colonic resection, radiation therapy to the abdomen or pelvis, colonic stricture, inflammatory bowel disease, polyposis syndrome, acute diverticulitis, lower gastrointestinal bleeding, or toxic megacolon. Patients were enrolled from one center in the Netherlands, one center in Israel, and one center in the United States between July 2013 and June 2014.

Six experienced gastroenterologists performed colonoscopies for this study. Study approval was obtained from the institutional review board or medical ethics committee at each participating center. This study was performed in compliance with the Declaration of Helsinki. Written, informed consent was obtained from all participants. This trial is registered with ClinicalTrials.gov (NCT01955122). All authors had access to the study data and reviewed and approved the final manuscript.

\section{Procedures}

Patients underwent same-day, back-to-back tandem colonoscopy examination performed by the same endoscopist. The randomized allocation determined whether patients underwent EndoRings colonoscopy immediately followed by standard colonoscopy or standard colonoscopy immediately followed by EndoRings colonoscopy.

Bowel preparation was done according to the standard protocols of the individual centers with $4 \mathrm{~L}$ split-dose polyethylene glycolbased solution or picosulfate solution. The quality of bowel preparation was assessed during colonoscopy using the Boston Bowel Preparation Scale (BBPS) $[23,24]$. Conscious sedation, which included a combination of midazolam and fentanyl, with or without propofol, was delivered to the patient by the gastroenterologist or an anesthesiologist.

All colonoscopies were performed with Olympus Evis Exera II 180 and 190 series or Pentax 3890 series colonoscopes. When the colonoscopy was being performed with the EndoRings, the device was attached to the distal tip of the same colonoscope as was used for the standard colonoscopy procedure. The colono-
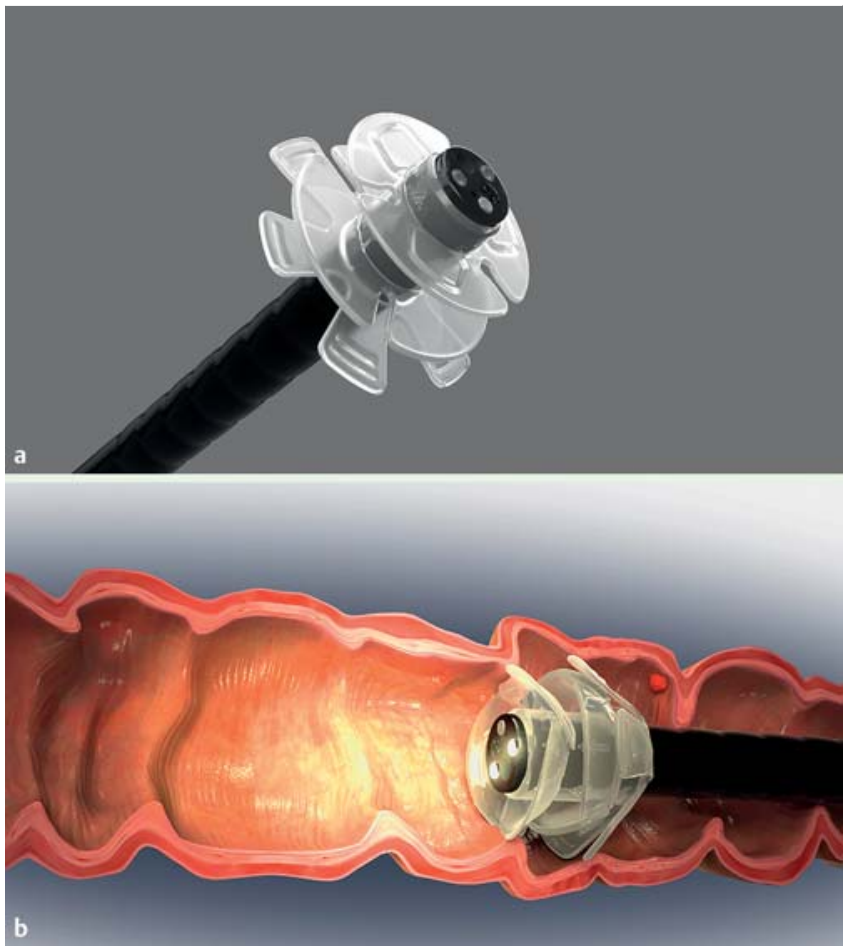

Fig. 1 The EndoRings device. a lllustration of the device attached to the distal end of a colonoscope. b Illustration showing how the device mechanically straightens the colonic folds during withdrawal of the colonoscope.

scope with the EndoRings was advanced to the cecum using a standard colonoscopic insertion technique.

Endoscopists were instructed to adhere to their usual withdrawal technique and to spend a minimum of 6 minutes withdrawing and examining the colonic mucosa. Cecal intubation time, withdrawal time, total procedure time, and time for pauses to allow polypectomies and biopsies to be performed were recorded by a research assistant using a stopwatch.

Polyps detected during the first procedure were immediately removed and sent for histology, as were polyps detected during the second colonoscopy. Diminutive (1-2 $\mathrm{mm}$ in size) rectal polyps with hyperplastic appearance were not removed and were not included in the analysis. When a polyp was detected, its estimated size and morphological appearance were reported by the endoscopist. Polyps located proximal to the splenic flexure were defined as proximally located colonic polyps; polyps located more distally were defined as distally located polyps. On the basis of histological examination, polyps were categorized as adenomatous, hyperplastic, or other. Adenomatous polyps were further categorized into tubular or (tubulo)villous lesions, with or without high grade dysplasia.

Patients were followed-up by telephone for adverse events at 24 hours and at 1 week after the colonoscopy.

\section{Randomization}

Randomization was performed using computer-generated randomization blocks ( $1: 1)$ with eight patients per block. The study site coordinator did not reveal the randomly assigned allocation until the start of the colonoscopy. Until that moment, patients and endoscopists were blinded to the allocation. 


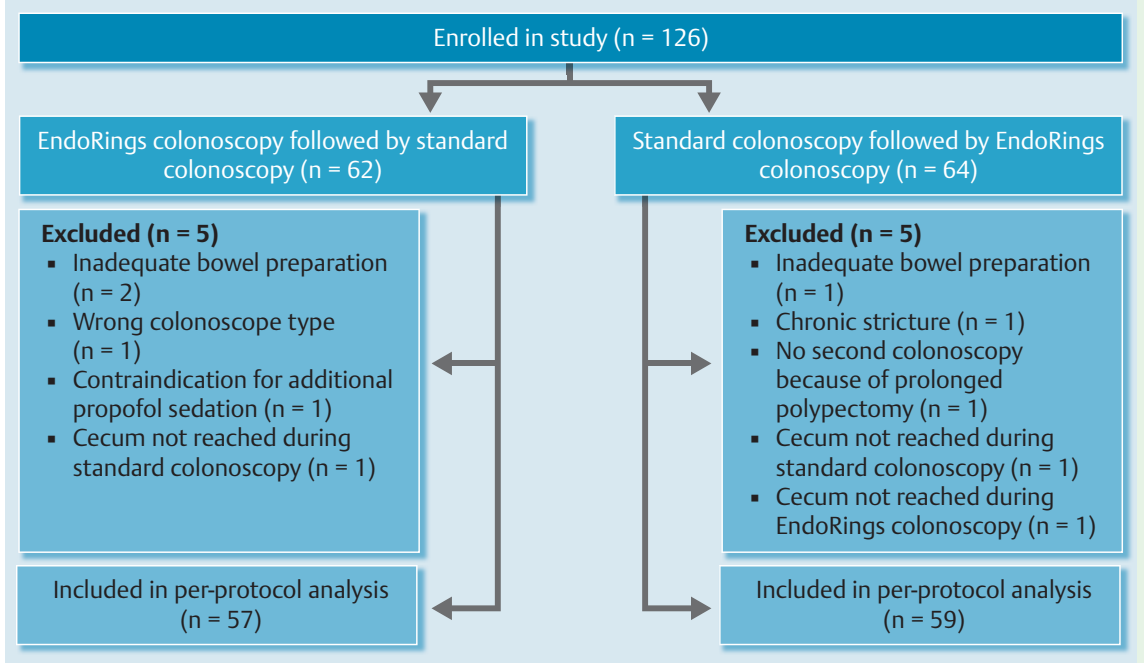

Fig. 2 Flow diagram of the patients enrolled in the CLEVER study.

Table 1 Number of tandem procedures performed per center and per endoscopist.

\begin{tabular}{|c|c|c|c|}
\hline Center & Procedures per center & Endoscopist number & Procedures per endoscopist \\
\hline Center A & 38 & 1 & 17 \\
\hline \multirow[t]{2}{*}{ GI Endoscopy Unit, Elisha Hospital, Haifa, Israel } & & 2 & 8 \\
\hline & & 3 & 13 \\
\hline Center B & 70 & 4 & 64 \\
\hline Department of Gastroenterology and Hepatology, & & 5 & 4 \\
\hline $\begin{array}{l}\text { University Medical Center Utrecht, Utrecht, The } \\
\text { Netherlands }\end{array}$ & & 6 & 2 \\
\hline Center C & 8 & 6 & 8 \\
\hline $\begin{array}{l}\text { Department of Medicine, Division of Gastroenterology } \\
\text { and Hepatology, Indiana University Hospital, Indianapolis, } \\
\text { Indiana, United States }\end{array}$ & & & \\
\hline
\end{tabular}

\section{Outcomes}

The primary endpoint of this study was the adenoma miss rate, which was defined as the number of adenomas detected during the second colonoscopy divided by the total number of adenomas detected during the first and second colonoscopy. Secondary outcomes included polyp miss rate, advanced adenoma miss rate, adenoma detection rate, false-negative first colonoscopy, impact on the recommended surveillance interval (according to the United States guidelines for colonoscopy surveillance after screening and polypectomy [3] and the European Union guidelines for quality assurance in colorectal cancer screening and diagnosis [1]), cecal intubation time, withdrawal time, total procedure time, and adverse events.

\section{Sample size calculation}

The required study sample size was calculated using a two-group chi-squared test with $80 \%$ power and 0.05 two-sided significance level and was based on an expected adenoma miss rate of $35 \%$ with standard colonoscopy and 10\% with EndoRings colonoscopy. This estimation was based on recent studies with techniques that improve visualization behind folds and flexures $[16,17]$. With an estimated mean number of adenomas per patient of 0.75 and a $10 \%$ dropout rate, the calculated total sample size required was 126 patients.

\section{Statistical analysis}

Descriptive statistics were calculated for all measured variables. For continuous variables, mean values and standard deviations (SDs) were calculated. Counts and percentages were calculated for categorical variables.

Two-sample Student's $t$ tests were used to compare age and BBPS between the study groups. Because patients acted as their own controls, paired $t$ tests were used to compare cecal intubation time, withdrawal time, time for polypectomies, and total procedure time between standard colonoscopy and colonoscopy with EndoRings. Pearson chi-squared and Fisher's exact tests were used to compare categorical variables, miss rates (per lesion analysis), and detection rates (per patient analysis) between the study groups.

Two-sided $P$ values below 0.05 were considered statistically significant. All statistical analyses were performed with IBM SPSS 22 (IBM Corp., Armonk, New York, USA).

\section{Results}

\section{Baseline characteristics}

A total of 126 patients were enrolled in this study and randomly assigned to undergo EndoRings colonoscopy followed by standard colonoscopy $(n=62)$ or standard colonoscopy followed by colonoscopy with EndoRings ( $n=64)$, of whom 116 (92.1\%) were included in the per-protocol analysis (see $\bullet$ Fig. 2 for flow diagram). The number of tandem procedures performed per center and per endoscopist are shown in $\bullet$ Table 1. Of the tandem colo- 
Table 2 Baseline characteristics of the per-protocol study population.

\begin{tabular}{|c|c|c|c|c|}
\hline Characteristics & Total & EndoRings colonoscopy first & Standard colonoscopy first & $P$ value $^{1}$ \\
\hline Patients & 116 & 57 & 59 & - \\
\hline Age, mean $\pm S D$, years & $58.7 \pm 9.2$ & $57.9 \pm 9.1$ & $59.6 \pm 9.3$ & 0.322 \\
\hline Female sex, n (\%) & $45(38.8 \%)$ & $16(28.1)$ & $29(49.2)$ & 0.020 \\
\hline BBPS, mean \pm SD, score & $7.8 \pm 1.1$ & $7.8 \pm 1.1$ & $7.8 \pm 1.1$ & 0.838 \\
\hline \multicolumn{5}{|l|}{ Indication, $\mathrm{n}(\%)$} \\
\hline Screening & $34(29.3 \%)$ & $17(29.8)$ & $17(28.8)$ & \multirow[t]{3}{*}{0.800} \\
\hline Surveillance & $40(34.5 \%)$ & $21(36.8)$ & $19(32.2)$ & \\
\hline Diagnostic & $42(36.2 \%)$ & $19(33.3)$ & $23(39.0)$ & \\
\hline \multicolumn{5}{|l|}{ Center } \\
\hline A & $38(32.8)$ & $18(31.6)$ & $20(33.9)$ & \multirow[t]{3}{*}{0.730} \\
\hline B & $70(60.3)$ & $36(63.2)$ & $34(57.6)$ & \\
\hline C & $8(6.9)$ & $3(5.3)$ & $5(8.5)$ & \\
\hline
\end{tabular}

SD, standard deviation; BBPS, Boston Bowel Preparation Scale.

${ }^{1}$ Continuous variables tested with Student's $t$ test; categorical variables tested with Pearson's chi-squared test.

Table 3 Adenomas ${ }^{1}$ detected and missed with EndoRings and standard colonoscopy.

\begin{tabular}{|c|c|c|c|}
\hline & EndoRings colonoscopy first & Standard colonoscopy first & $P$ value $^{\dagger 2}$ \\
\hline \multicolumn{4}{|l|}{ Adenomas detected, $\mathrm{n}$} \\
\hline First procedure & 60 & 30 & \\
\hline Second procedure ${ }^{3}$ & 7 & 28 & \\
\hline Total & 67 & 58 & \\
\hline Adenoma miss rate in first procedure $(95 \% \mathrm{Cl}), \%$ & $10.4(2.9-18.0)$ & $48.3(35.0-61.2)$ & $<0.001$ \\
\hline \multicolumn{4}{|l|}{ Adenoma miss rates by characteristics, $\mathrm{n}(\%)$} \\
\hline Proximal & $5(10.6 \%)$ & $18(58.1 \%)$ & $<0.001$ \\
\hline Distal & $2(10.0 \%)$ & $10(37.0 \%)$ & 0.047 \\
\hline $1-5 \mathrm{~mm}$ & $7(13.5 \%)$ & $26(54.2 \%)$ & $<0.001$ \\
\hline $6-9 \mathrm{~mm}$ & $0(0.0 \%)$ & $2(33.3 \%)$ & 0.125 \\
\hline$\geq 10 \mathrm{~mm}$ & $0(0.0 \%)$ & $0(0.0 \%)$ & - \\
\hline Pedunculated & $1(5.3 \%)$ & $1(14.3 \%)$ & 0.474 \\
\hline Sessile & $6(12.8 \%)$ & $22(50.0 \%)$ & $<0.001$ \\
\hline Flat & $0(0.0 \%)$ & $5(71.4 \%)$ & 0.375 \\
\hline Tubular & $7(11.1 \%)$ & $28(50.9 \%)$ & $<0.001$ \\
\hline (Tubulo)villous & $0(0.0 \%)$ & $0(0.0 \%)$ & - \\
\hline Low grade dysplasia ${ }^{4}$ & $7(10.8 \%)$ & $27(50.0 \%)$ & $<0.001$ \\
\hline High grade dysplasia & $0(0.0 \%)$ & $0(0.0 \%)$ & - \\
\hline \multicolumn{4}{|l|}{ Patients with at least one adenoma detected } \\
\hline First procedure & $28 / 57$ & $17 / 59$ & \\
\hline Second procedure ${ }^{3}$ & $4 / 57$ & $17 / 59$ & \\
\hline Total & $28 / 57$ & $27 / 59$ & \\
\hline Adenoma detection rate in first procedure $(95 \% \mathrm{Cl}), \%$ & $49.1(35.7-62.5)$ & $28.8(16.9-40.7)$ & 0.025 \\
\hline False-negative first procedure ( $95 \% \mathrm{Cl}), \%$ & 0.0 & $16.9(7.1-26.8)$ & 0.001 \\
\hline
\end{tabular}

$\mathrm{Cl}$, confidence interval.

1 Including tubular adenomas and (tubulo)villous adenomas.

${ }^{2}$ Tested with Pearson's chi-squared test.

${ }^{3}$ Cross-over design with removal of lesions during the first procedure.

${ }^{4}$ Grade of dysplasia unknown in five of the adenomas detected during the first procedure and in one detected during the second procedure.

noscopy procedures, 38 (32.8\%) were performed in center A, 70 $(60.3 \%)$ in center $\mathrm{B}$, and $8(6.9 \%)$ in center C. One endoscopist (number 6) performed procedures in both centers $B$ and $C$.

Patients had a mean age of $58.7 \pm 9.2$ years and $38.8 \%$ were women. The indications for colonoscopy were screening $(n=34 ; 29.3$ $\%$ ), surveillance $(n=40 ; 34.5 \%)$, and diagnostic evaluation ( $n=$ 42 ; $36.2 \%)$. Mean age $(P=0.322)$ and BBPS scores $(P=0.838)$ were not significantly different statistically between the two study groups ( $\bullet$ Table 2 ). However, the group that underwent standard colonoscopy first included significantly more women $(P=0.020)$ compared with the group that underwent EndoRings colonoscopy first.

\section{Adenoma miss rate}

In patients who underwent EndoRings colonoscopy first, 60 adenomas were detected in 28 patients, while during the secondpass standard colonoscopy an additional seven adenomas were found in four patients ( Table 3 ). In patients who underwent standard colonoscopy first, 30 adenomas were detected in 17 patients with standard colonoscopy, while the second-pass colonoscopy with EndoRings identified 28 additional adenomas in 17 patients. Therefore, the adenoma miss rate was significantly lower $(P<0.001)$ with EndoRings colonoscopy (10.4\%) compared with standard colonoscopy (48.3\%). 
Table 5 Polyps ${ }^{1}$ detected and missed with EndoRings and standard colonoscopy.

\begin{tabular}{|c|c|c|c|}
\hline & EndoRings colonoscopy first & Standard colonoscopy first & $P$ value ${ }^{2}$ \\
\hline \multicolumn{4}{|l|}{ Polyps detected } \\
\hline First procedure & 110 & 50 & \\
\hline Second procedure ${ }^{3}$ & 11 & 56 & \\
\hline Total & 121 & 106 & \\
\hline Polyp miss rate for first procedure, $\%$ & $9.1(3.9-14.3)$ & $52.8(43.2-62.5)$ & $<0.001$ \\
\hline \multicolumn{4}{|l|}{ Polyp miss rates by characteristics, $n$ (\%) } \\
\hline Proximal & $7(10.8 \%)$ & $28(62.2 \%)$ & $<0.001$ \\
\hline Distal & $4(7.1 \%)$ & $28(45.9 \%)$ & $<0.001$ \\
\hline $1-5 \mathrm{~mm}$ & $11(10.7 \%)$ & $53(57.6 \%)$ & $<0.001$ \\
\hline $6-9 \mathrm{~mm}$ & $0(0.0 \%)$ & $3(33.3 \%)$ & 0.074 \\
\hline$\geq 10 \mathrm{~mm}$ & $0(0.0 \%)$ & $0(0.0 \%)$ & - \\
\hline Pedunculated & $2(6.9 \%)$ & $5(33.3 \%)$ & 0.036 \\
\hline Sessile & $9(10.6 \%)$ & $41(52.6 \%)$ & $<0.001$ \\
\hline Flat & $0(0.0 \%)$ & $10(76.9 \%)$ & 0.003 \\
\hline Hyperplastic & $4(8.0 \%)$ & $28(59.6 \%)$ & $<0.001$ \\
\hline Adenoma & $7(10.4 \%)$ & $28(48.3)$ & $<0.001$ \\
\hline Serrated & $0(0.0 \%)$ & $0(0.0 \%)$ & - \\
\hline Other & $0(0.0 \%)$ & $0(0.0 \%)$ & - \\
\hline \multicolumn{4}{|l|}{ Patients with at least one polyp detected } \\
\hline First procedure & $39 / 57$ & $24 / 59$ & \\
\hline Second procedure ${ }^{3}$ & $7 / 57$ & $32 / 59$ & \\
\hline Total & $39 / 57$ & $39 / 59$ & \\
\hline Polyp detection rate for first procedure $(95 \% \mathrm{Cl})$, \% & $68.4(56.0-80.9)$ & $40.7(27.8-53.6)$ & 0.003 \\
\hline False-negative first procedure ( $95 \% \mathrm{Cl}), \%$ & 0.0 & $25.4(14.0-36.9)$ & $<0.001$ \\
\hline
\end{tabular}

$\mathrm{Cl}$, confidence interval.

${ }^{1}$ Including hyperplastic polyps, tubular adenomas, (tubulo)villous adenomas, serrated polyps, lipomas, leiomyomas and inflammatory polyps

2 Tested with Pearson's chi-squared test.

${ }^{3}$ Cross-over design with removal of lesions during the first procedure.

Adenoma miss rates per center are shown in $\bullet$ Table 4 (available online). In center B, adenoma miss rates with standard and EndoRings colonoscopy were $31.8 \%$ versus $14.9 \%$ respectively $(P=$ 0.103 ); in both centers $A$ and $C$, adenoma miss rates were $58.3 \%$ versus $0.0 \%$ respectively.

\section{Polyp miss rate}

EndoRings colonoscopy identified 110 polyps in 39 patients during first-pass examinations. With second-pass standard colonoscopy, an additional 11 polyps were found in seven patients ( $\bullet$ Table 5). In patients who underwent standard colonoscopy first, 50 polyps were found in 24 patients during the first-pass examinations, while during the second-pass colonoscopies using EndoRings an additional 56 polyps were found in 32 patients. Therefore, the polyp miss rate was significantly lower $(P<0.001)$ with EndoRings colonoscopy (9.1\%) compared with standard colonoscopy $(52.8 \%)$.

\section{Adenoma and polyp detection rate}

Adenoma detection rate of the first-pass colonoscopy was higher $(P=0.025)$ with EndoRings (28 of 57 patients; $49.1 \%)$ than with standard colonoscopy (17 of 59 patients; $28.8 \%$ ). Moreover, in the group that underwent standard colonoscopy first, 10 patients had no adenomas detected during first-pass colonoscopy but were found to have adenomas during the second-pass colonoscopy with EndoRings, resulting in a false-negative colonoscopy rate for adenomas of $16.9 \%$. In contrast, the false-negative colonoscopy rate of EndoRings colonoscopy was $0 \%(P=0.001)$. Furthermore, the polyp detection rate with EndoRings (68.4\%) was significantly higher $(P=0.003)$ than with standard colonoscopy (40.7\%).

\section{Characteristics of missed adenomas}

The miss rate of proximally located adenomas was significantly lower $(P<0.001)$ with EndoRings (5 of $47 ; 10.6 \%)$ compared with standard colonoscopy ( 18 of $31 ; 58.1 \%$ ). Most of the missed adenomas were $1-5 \mathrm{~mm}$ in size (100\% for EndoRings and $92.9 \%$ for standard colonoscopy) and these small adenomas were less frequently missed with EndoRings ( 7 of $52 ; 13.5 \%$ ) than with standard colonoscopy ( 26 of $48 ; 54.2 \% ; P<0.001$ ). There were no differences in the miss rates for adenomas that were $6-9 \mathrm{~mm}(P$ $=0.125)$, pedunculated $(P=0.474)$, or flat $(P=0.375)$. However, the miss rate of sessile adenomas was significantly lower $(P<0.001)$ with EndoRings ( 6 of $47 ; 12.8 \%$ ) than with standard colonoscopy (22 of $44 ; 50.0 \%$ ).

All adenomas missed with standard or EndoRings colonoscopy were tubular adenomas smaller than $10 \mathrm{~mm}$ and none showed histological evidence of high grade dysplasia, meaning that no advanced adenomas were missed with EndoRings or standard colonoscopy. There were no serrated polyps missed with EndoRings or standard colonoscopy.

\section{Impact on surveillance intervals}

The impact of the second-pass colonoscopy using EndoRings and standard colonoscopy is shown in Table e6 (available online). Of the 59 patients who underwent standard colonoscopy first, in eight patients (13.6\%) the recommended surveillance interval, according to current US guidelines, changed from 10 years to 5 years and in three $(5.1 \%)$ the recommended surveillance interval changed from 5 years to 3 years after the secondpass colonoscopy with EndoRings. In contrast, in only two of the 57 patients (3.5\%) who underwent EndoRings colonoscopy first was the recommended surveillance interval shortened after the 


\begin{tabular}{|llll|}
\hline $\begin{array}{l}\text { Time endpoints, } \\
\text { mean } \pm \text { SD, minutes }\end{array}$ & EndoRings colonoscopy & Standard colonoscopy & $\boldsymbol{P}_{\text {value }}{ }^{\mathbf{1}}$ \\
\hline Cecum intubation time & $9.3 \pm 7.3$ & $8.4 \pm 5.6$ & 0.142 \\
\hline Withdrawal time & $7.4 \pm 1.9$ & $7.2 \pm 2.2$ & 0.286 \\
\hline Time spent on polypectomies & $3.5 \pm 4.3$ & $1.8 \pm 4.5$ & 0.001 \\
\hline Time per polypectomy & $3.0 \pm 2.5$ & $2.8 \pm 2.1$ & 0.697 \\
\hline Total procedure time & $21.6 \pm 8.9$ & $18.5 \pm 8.2$ & 0.001 \\
\hline
\end{tabular}

Table 7 Procedural time endpoints for EndoRings and standard colonoscopy

SD, standard deviation.

1 Tested with paired $t$ test.

second-pass standard colonoscopy. This difference was significant $(P=0.013)$.

When applying the European guidelines, the recommend surveillance interval was shortened in three patients (5.3\%) who underwent EndoRings colonoscopy first and in five patients (8.5\%) who underwent standard colonoscopy first $(P=0.617)$.

\section{Time endpoints}

The mean cecal intubation time, withdrawal time, and total procedure time are shown in $\bullet$ Table 7. The mean \pm SD cecal intubation time was shorter with standard colonoscopy $(8.4 \pm 5.6 \mathrm{~min}-$ utes) compared with EndoRings colonoscopy $(9.3 \pm 7.3$ minutes $)$ but this difference was not statistically significant $(P=0.142)$. Mean \pm SD withdrawal time was comparable $(P=0.286)$ between standard (7.2 \pm 2.2 minutes) and EndoRings colonoscopy (7.4 \pm 1.9 minutes), including when this was limited to the first-pass colonoscopies $(7.0 \pm 2.1$ vs. $7.8 \pm 2.1$ minutes; $P=0.055)$.

Total procedure time was approximately 3 minutes longer with EndoRings colonoscopy (21.6 \pm 8.9 minutes) compared with standard colonoscopy (18.5 \pm 8.2 minutes), which was because more time was required for the additional polypectomies ( $3.5 \pm$ 4.3 minutes vs. $1.8 \pm 4.5$ minutes; $P=0.001$ ). The time per polypectomy was similar with EndoRings and standard colonoscopy (3.0 \pm 2.5 minutes vs. $2.8 \pm 2.1$ minutes; $P=0.697$ ).

\section{Adverse events}

No adverse events related to the EndoRings device occurred during the conduct of this study. However, in one patient the cecum could not be intubated in the EndoRings colonoscopy and in two other patients the cecum was not intubated in the standard colonoscopy. In a fourth patient, a second procedure with the EndoRings was not performed because of a prolonged polypectomy requiring the placement of multiple clips during the first procedure with a standard colonoscope.

\section{Discussion}

$\nabla$

Although colonoscopy is currently the preferred method of detection for (pre)cancerous lesions in the colorectum [1-4], it is increasingly being recognized as an imperfect method of examination because of its inability to visualize the entire mucosal surface, particularly the proximal aspects of folds and flexures $[12$, 13].

In the present randomized, tandem colonoscopy study, we showed that significantly fewer adenomas and polyps are missed with EndoRings colonoscopy and that both the adenoma and polyp detection rates are significantly higher compared with standard colonoscopy. Although we were unable to demonstrate a statically significant difference in missed advanced adenomas, a substantial number of flat and proximally located lesions were missed with standard colonoscopy while such lesions were less frequently missed with EndoRings colonoscopy. Furthermore, the recommended colonoscopy surveillance interval was more frequently shortened after the second procedure with EndoRings than with the standard procedure because of additional adenomas being detected.

The improved detection of polyps and adenomas with the EndoRings is provided by three circular rows of flexible siliconerubber rings that engage and mechanically straighten the colonic folds during withdrawal. The EndoRings additionally improves visualization of the total colonic surface area by keeping the distal tip of the colonoscope centered in the colonic lumen. Colonoscopy with the EndoRings does not interfere with the normal washing, suctioning, and therapeutic capabilities of the colonoscope and does not block parts of the camera view as some other devices may do [25].

Although it is difficult to make direct comparisons between studies, the results of the present study indicate that the effect of the EndoRings on polyp and adenoma miss rates is in the upper range of the effects of other devices or techniques designed to improve visualization of the colonic mucosa, such as cap-assisted colonoscopy [26,27], virtual chromoendoscopy [20], and Third Eye Retroscope colonoscopy [17].

The recently developed Endocuff seems to have features comparable to the EndoRings, with both being add-on devices that are designed to flatten the mucosal folds during withdrawal [28]. In a recent randomized, controlled trial, the Endocuff was shown to significantly improve both polyp ( $56 \%$ vs. $42 \%$ ) and adenoma detection rates (36\% vs. $28 \%$ ) as compared with standard colonoscopy [29].

Another recently introduced technology is FUSE colonoscopy. This novel colonoscope has three cameras incorporated in the tip that enlarge the field of view up to $330^{\circ}$ compared with the $170^{\circ}$ of a conventional standard colonoscope. The advantage of the FUSE colonoscope has recently been demonstrated in the FUSE study, a randomized, back-to-back tandem study in which significantly lower adenoma miss rates were found when using FUSE colonoscopy compared with standard colonoscopy ( $7 \%$ vs. $41 \% ; P<0.0001$ ) [16]. These results closely resemble the findings of our study.

Although maneuverability may be expected to be easier with the FUSE colonoscope, the advantage of the EndoRings is that it is compatible with the currently used standard colonoscopes, making it a cheaper alternative that can be easily applied in health care systems with budget constraints. Nonetheless, insertion of the colonoscope when using EndoRings may be more challenging in patients with strictures, previous bowel surgery, or severe diverticulosis because the flexible rings of the EndoRings may cause some resistance during advancement of the colonoscope. This is probably why we found a slightly, but not significantly, longer cecal intubation time with EndoRings colonoscopy; how- 
ever, being less than a minute longer, this difference is not likely to be clinically relevant.

The total procedure time was 3 minutes longer with EndoRings colonoscopy compared with standard colonoscopy, which was because of the extra time required for polypectomies. The time per polypectomy was similar when EndoRings was compared with standard colonoscopy. The tip control during polypectomy was not reported to be a limitation by the performing endoscopists. In contrast, some endoscopists reported a benefit of using the EndoRings, in that it stabilized the tip of the colonoscope in the lumen. Nonetheless, the longer total procedure time and shortened surveillance interval should be balanced against the additional diagnostic yield with EndoRings colonoscopy. In our view, the latter is preferable if the aim of colonoscopy is to reduce the risk of developing colorectal cancer.

The higher number of adenomas that were removed significantly affects the recommended colonoscopy surveillance interval, as was demonstrated in this study. The clinical relevance of this may be argued as, for the most part, this was because more small sessile adenomas were found. However, we also demonstrated that the miss rate of proximally located adenomas was significantly lower and no flat adenomas were missed with EndoRings colonoscopy. This is highly relevant when it is considered that colonoscopy is less effective in preventing colorectal cancer in the proximal colon particularly [30 - 33], largely as a result of missed (pre)cancerous lesions often with flat morphology [8,9].

Our study has some limitations. First, the study was powered to perform a per-lesion analysis and not to detect a difference in adenoma or polyp detection rates (per-patient analysis). Nevertheless, and importantly, significantly higher adenoma and polyp detection rates were found with EndoRings colonoscopy. Second, the study was not powered to detect differences between groups with an indication for screening, surveillance, or diagnostic colonoscopy. This may be important as some subgroups of patients might benefit more from the use of add-on devices or technologies during colonoscopy than others.

Third, the same endoscopist performed both procedures and was therefore not blinded to the findings of the first-pass procedures and may not have used the same effort during both colonoscopy procedures when trying to detect adenomas. This may to some extent have favored EndoRings colonoscopy. However, investigator bias may also not be fully prevented when two different endoscopists perform the back-to-back colonoscopies. Moreover, choosing one endoscopist for both procedures has the advantage of preventing bias due to differences in withdrawal technique and overall adenoma detection rates between endoscopists. In other words, the single endoscopist acts as his/her own control. Fourth, procedures were not evenly distributed among the study centers with most procedures $(60.3 \%)$ being performed in center B. In this center, the adenoma miss rate with standard colonoscopy was lower, while the miss rate with EndoRings colonoscopy was higher compared with the other two centers that included significantly fewer participants. This finding may imply a type I error in the two centers that included fewer participants. The total adenoma miss rates that we found should therefore be interpreted with caution and may, in reality, be similar to the miss rates found in center $B$. This is also supported by the findings of previous back-to-back colonoscopy studies in which adenoma miss rates of standard colonoscopy were lower.

Fifth, the endoscopists in this study were instructed to apply a minimum withdrawal time of 6 minutes but no restrictions were given for maximum withdrawal time. However, the mean withdrawal times were similar for standard and EndoRings colonoscopy.

Finally, 10 patients were not included in the analysis because of protocol violations. In two patients undergoing standard colonoscopy first, the advancement of the colonoscope (in one case with standard and in another with EndoRings colonoscopy) was not possible because of a narrow sigmoid as a consequence of diverticulosis. In a further patient it was decided not to perform a second colonoscopy with the EndoRings because of a colonic stricture. In another patient, the cecum was not intubated during standard colonoscopy, but the cecum was successfully intubated with the EndoRings. It should be noted that the manufacturer recently developed an EndoRings that fits onto a pediatric colonoscope for patients with a narrow colon.

No adverse events related to the EndoRings occurred during the conduct of this study.

In conclusion, the results of this multicenter, randomized, backto-back, tandem colonoscopy study demonstrate that, as compared with standard colonoscopy, colonoscopy with the EndoRings device significantly reduces the adenoma and polyp miss rates. The simple and easy-to-use design and the minimal impact on cecal intubation time of this add-on device may help to improve the efficacy of screening and surveillance colonoscopies.

The next step in the evaluation of this device should investigate adenoma detection rates in a parallel, randomized, controlled trial including more endoscopists and more study patients. In addition, it will be interesting to investigate whether specific histological types of polyps or polyp sizes are more frequently found with EndoRings colonoscopy.

Competing interests: This study was funded by EndoAid Ltd., Caesarea, Israel. Drs. Gralnek, Siersema, Rex, and Segol were consultants for EndoAid Ltd. during the conduct of this study. Dr. Segev is an employee of EndoAid Ltd. Drs. Dik, Suissa, Belderbos, Moons, and Domanov have nothing to disclose.

\section{Institutions}

${ }^{1}$ Department of Gastroenterology and Hepatology, University Medical Center Utrecht, Utrecht, The Netherlands

${ }^{2}$ Institute of Gastroenterology and Liver Diseases, Ha’Emek Medical Center, Afula, Israel

${ }^{3}$ GI Endoscopy Unit, Elisha Hospital, Haifa, Israel

${ }^{4}$ Rappaport Family Faculty of Medicine, Technion-Israel Institute of Technology, Haifa, Israel

${ }^{5}$ Department of Gastroenterology, Lady Davis Carmel Medical Center, Haifa, Israel

${ }^{6}$ Department of Gastroenterology, Rambam Health Care Campus, Haifa, Israel ${ }^{7}$ EndoAid Ltd., Caesarea, Israel

${ }^{8}$ Department of Medicine, Division of Gastroenterology and Hepatology, Indiana University Hospital, Indianapolis, IN, United States

\section{References}

1 Atkin WS, Valori R, Kuipers EJ et al. European guidelines for quality assurance in colorectal cancer screening and diagnosis. First Edition-Colonoscopic surveillance following adenoma removal. Endoscopy 2012; 44: SE151-SE163

2 Lieberman DA, Weiss DG, Bond JH et al. Use of colonoscopy to screen asymptomatic adults for colorectal cancer. Veterans Affairs Cooperative Study Group 380. NEJM 2000; 343: 162-168

3 Lieberman DA, Rex DK, Winawer SJ et al. Guidelines for colonoscopy surveillance after screening and polypectomy: a consensus update by the US Multi-Society Task Force on Colorectal Cancer. Gastroenterology 2012; 143: $844-857$

4 Regula J, Rupinski M, Kraszewska E et al. Colonoscopy in colorectal-cancer screening for detection of advanced neoplasia. NEJM 2006; 355: $1863-1872$

5 Baxter NN, Goldwasser MA, Paszat LF et al. Association of colonoscopy and death from colorectal cancer. Ann Intern Med 2009; 150: 1-8 
6 Cottet V, Jooste V, Fournel I et al. Long-term risk of colorectal cancer after adenoma removal: a population-based cohort study. Gut 2012; 61: $1180-1186$

7 Kahi CJ, Imperiale TF, Juliar BE et al. Effect of screening colonoscopy on colorectal cancer incidence and mortality. Clin Gastroenterol Hepatol 2009; 7: 770-775

8 le Clercq CM, Bouwens MW, Rondagh EJ et al. Postcolonoscopy colorectal cancers are preventable: a population-based study. Gut 2014; 63: 957-963

9 Robertson DJ, Lieberman DA, Winawer SJ et al. Colorectal cancers soon after colonoscopy: a pooled multicohort analysis. Gut 2013; 63: 949956

10 Zauber AG, Winawer SJ, O'Brien MJ et al. Colonoscopic polypectomy and long-term prevention of colorectal-cancer deaths. NEJM 2012; 366: 687-696

11 Pohl H, Robertson DJ. Colorectal cancers detected after colonoscopy frequently result from missed lesions. Clin Gastroenterol Hepatol 2010; 8: $858-864$

12 Pickhardt PJ, Choi JR, Hwang I et al. Computed tomographic virtual colonoscopy to screen for colorectal neoplasia in asymptomatic adults. NEJM 2003; 349: 2191 - 2200

13 Pickhardt PJ, Nugent PA, Mysliwiec PA et al. Location of adenomas missed by optical colonoscopy. Ann Intern Med 2004; 141: 352 - 359

14 East JE, Saunders BP, Burling D et al. Surface visualization at CT colonography simulated colonoscopy: effect of varying field of view and retrograde view. Am J Gastroenterol 2007; 102: 2529-2535

15 van Rijn JC, Reitsma JB, Stoker J et al. Polyp miss rate determined by tandem colonoscopy: a systematic review. Am J Gastroenterol 2006; 101 : $343-350$

16 Gralnek IM, Siersema PD, Halpern Z et al. Standard forward-viewing colonoscopy versus full-spectrum endoscopy: an international, multicentre, randomised, tandem colonoscopy trial. Lancet Oncol 2014; 15: $353-360$

17 Leufkens AM, DeMarco DC, Rastogi A et al. Effect of a retrograde-viewing device on adenoma detection rate during colonoscopy: the TERRACE study. Gastrointest Endosc 2011; 73: 480-489

18 Rex DK, Petrini JL, Baron TH et al. Quality indicators for colonoscopy. Gastrointest Endosc 2006; 63: S16-S28

$19 \mathrm{Ng} \mathrm{SC}$, Tsoi KK, Hirai HW et al. The efficacy of cap-assisted colonoscopy in polyp detection and cecal intubation: a meta-analysis of randomized controlled trials. Am J Gastroenterol 2012; 107: 1165 - 1173
20 Pasha SF, Leighton JA, Das A et al. Comparison of the yield and miss rate of narrow band imaging and white light endoscopy in patients undergoing screening or surveillance colonoscopy: a meta-analysis. Am J Gastroenterol 2012; 107: 363-370

21 Dik VK, Moons LM, Siersema PD. Endoscopic innovations to increase the adenoma detection rate during colonoscopy. World J Gastroenterol 2014; 20: 2200 - 2211

22 Rex DK, Bond JH, Winawer S et al. Quality in the technical performance of colonoscopy and the continuous quality improvement process for colonoscopy: recommendations of the U.S. Multi-Society Task Force on Colorectal Cancer. Am J Gastroenterol 2002; 97: 1296 - 1308

23 Calderwood AH, Jacobson BC. Comprehensive validation of the Boston Bowel Preparation Scale. Gastrointest Endosc 2010; 72: 686 - 692

24 Lai EJ, Calderwood AH, Doros G et al. The Boston bowel preparation scale: a valid and reliable instrument for colonoscopy-oriented research. Gastrointest Endosc 2009; 69: 620-625

25 Mamula P, Tierney WM, Banerjee $S$ et al. Devices to improve colon polyp detection. Gastrointest Endosc 2011; 73: 1092 - 1097

26 Hewett DG, Rex DK. Cap-fitted colonoscopy: a randomized, tandem colonoscopy study of adenoma miss rates. Gastrointest Endosc 2010; 72: $775-781$

27 Matsushita M, Hajiro K, Okazaki K et al. Efficacy of total colonoscopy with a transparent cap in comparison with colonoscopy without the cap. Endoscopy 1998; 30: 444-447

28 Lenze F, Beyna T, Lenz P et al. Endocuff-assisted colonoscopy: a new accessory to improve adenoma detection rate? Technical aspects and first clinical experiences Endoscopy 2014; 46: 610-614

29 Biecker E, Floer M, Heinecke A et al. Novel Endocuff-assisted colonoscopy significantly increases the polyp detection rate: a randomized controlled trial. J Clin Gastroenterol 2015; 49: 413-418

30 Bressler B, Paszat LF, Vinden C et al. Colonoscopic miss rates for rightsided colon cancer: a population-based analysis. Gastroenterology 2004; 127: $452-456$

31 Singh $H$, Nugent $Z$, Demers AA et al. The reduction in colorectal cancer mortality after colonoscopy varies by site of the cancer. Gastroenterology 2010; 139: $1128-1137$

32 Brenner $H$, Hoffmeister $M$, Arndt Vet al. Protection from right- and leftsided colorectal neoplasms after colonoscopy: population-based study. J Natl Cancer Inst 2010; 102: 89-95

33 Baxter NN, Warren JL, Barrett MJ et al. Association between colonoscopy and colorectal cancer mortality in a US cohort according to site of cancer and colonoscopist specialty. J Clin Oncol 2012; 30: 2664-2669

Table e4, e6

online content viewable at:

http://dx.doi.org/10.1055/s-0034-1392421 